\title{
A Method for Determining Classroom Seating Arrangements by Using Bioinformatics
}

\author{
Kazuhiro Shin-ike ${ }^{1, *}$ and Hitoshi Iima ${ }^{2}$ \\ ${ }^{1}$ Maizuru National College of Technology, 234, Shiraya, Maizuru, Kyoto 625-8511, Japan \\ shinike@maizuru-ct.ac.jp \\ ${ }^{2}$ Kyoto Institute of Technology, Matsugasaki, Sakyo-ku, Kyoto 606-8585, Japan \\ iima@kit.ac.jp
}

Students in a classroom often feel an uncomfortable situation in classroom seating arrangements. This study focuses on the effects of classroom seating arrangements changes on motivation of learning and on interaction between students. In this study we inspect the classroom seating arrangements and the tendency of behavior of students by the difference of their seats. We also propose a method for determining the classroom seating arrangements by using a genetic algorithm to improve the learning environment. We carry out a questionnaire about classroom seating arrangements. We also inspect the effects of human relationship between students, flexibility of each student for classroom seating arrangements and behaviors of students of choosing the seats. A model of the classroom seating arrangements is constructed by using the results of the questionnaire and an analysis of an observation of behaviors between students.

In order to determine the optimal classroom seating arrangements, a genetic algorithm is applied on the basis of the questionnaire results and the analysis of the observation of behaviors between students. Our optimization problem is to determine the combination of classroom seating arrangements in such a way that the minimum of fitness rates of all students is as large as possible.

Two kinds of calculation results are obtained in the case where final generations are ten thousand and hundred thousand. Experiments in the classroom are carried out by using two kinds of classroom seating arrangements. We compare our proposed classroom seating arrangements with traditional one that is determined by using students ID numbers or intentions of the students and the homeroom teacher.

From the questionnaire of the classroom seating arrangements, it is found that satisfaction of each student for our proposed method is higher than the traditional classroom seating arrangements. It is also found from another questionnaire that each student is actually comfortable in the classroom because of the relations between other students. It is confirmed from the experimental results that the proposed method was effective. This proposed method can determine the classroom seating arrangements by a simple process in a short time. When teachers make classroom seating arrangements to improve the effect of learning, this research can be used effectively for an evaluation and an improvement of teaching.

${ }^{*}$ Corresponding author. 